\title{
Mechanical characterization of Ti-6Al-4V titanium alloy at multiple length scales using spherical indentation stress-strain measurements
}

\section{Authors:}

Jordan S. Weaver ${ }^{\mathrm{a}, \mathrm{b}}$ and Surya R. Kalidindi ${ }^{\mathrm{b} *}$

${ }^{a}$ Center for Integrated Nanotechnologies, Los Alamos National Laboratory, Los Alamos, NM, USA

${ }^{\mathrm{b}}$ George W. Woodruff School of Mechanical Engineering, Georgia Institute of Technology, Atlanta, GA, USA

* Corresponding author. Tel.: +1 4043852886.

E-mail address: surya.kalidindi@me.gatech.edu (S.R. Kalidindi).

URL: http://mined.gatech.edu/

\begin{abstract}
Recent advances in spherical indentation stress-strain protocols and analyses have demonstrated the capability for measuring reliably the local mechanical responses in polycrystalline metal samples at different length scales, ranging from sub-micron (regions within individual grains) to several hundreds of microns (regions covering several grains). These recent advances have now made it possible to study systematically the mechanical behavior of a single material system at different length scales, with tremendous potential to obtain new insights into the role of individual phases, interfaces, and other microscale constituents on the macroscale mechanical response of the material. In this paper, we report spherical indentation stress-strain measurements with different indenter sizes (microns to millimeters) on Ti-6Al-4V (Ti-64) which capture the mechanical response of single phase alpha-Ti-64, single colony (alpha-beta), few colonies, and many colonies of Ti-64. The results show that the average mechanical response (indentation modulus and yield strength) from multiple indentations remains relatively unchanged from single phase alpha to many colonies of Ti-64, while the variance in the response decreases with indenter size. The work-hardening response in indentation tests follows a similar
\end{abstract}


behavior up to indentation zones of many colonies, which shows significantly higher work hardening rates.

Keywords: Hertzian indentation, Ti-64, multi-scale, nanoindentation, microindentation

\section{Introduction}

Titanium alloys exhibit high strength to weight ratios with excellent corrosion resistance and fatigue properties typically used in the aerospace industry $[1,2]$. Their microstructures vary significantly and often contain distinct distributions of $\alpha$ (hcp crystal) and $\beta$ (bcc crystal) phases at different length scales. Microstructure sensitive models are critically needed to aid in the development of new, stronger, more fatigue resistant titanium alloys [3-7] and understand the relationships between microstructure and properties. These models (e.g., [8-12]) rely on experiments for parameter calibration. Typically, the process utilizes bulk mechanical tests (e.g., uniaxial tension and compression tests). It is sensible to isolate the mechanical response of individual constituents at different structural length scales in studies of complex microstructures that exhibit structural hierarchy (distinct structures at different length scales) in order to improve these models. Additionally, any experimental protocols aimed at achieving this type of characterization must be high throughput, given the number of materials, microstructural features, and length scales involved. Indentation is the practical choice, given the ease and simplicity of doing experiments.

Indentation testing has long been used as a mechanical characterization tool; however, measures like hardness are limited in nature to qualitative conclusions. This is in part due to the protocols themselves (e.g., hardness often depends on the indenter geometry and load applied). One of the things lost in Tabor's [13] pioneering indentation work (where the relation of hardness to uniaxial strength is rooted) was that the spherical indentation response can be 
converted into an effective stress-strain response by normalizing the load by the cross-sectional contact area and defining an effective measure of strain (e.g., a/R). The protocols for spherical indentation stress-strain curves have undergone significant updates since Tabor, with the advent of better instrumentation and understanding [14-18]. The work by Kalidindi and Pathak [18] diverges from the Tabor's definition of indentation strain and previously used definitions of contact radius. These differences in protocols were evaluated critically with FEM simulations $[19,20]$. It was shown that the protocols of Kalidindi and Pathak [18] use a definition of contact radius that is consistent with Hertz's theory [21,22] (the basis for contact mechanics) for elastic loading and unloading (after plasticity occurs). Additionally, it was argued that the new definition of indentation strain is (i) a more physically reasonable definition (allows interpretation as change in length over length), (ii) provides a more reasonable average strain of the heterogeneous strain field under the indenter, and (iii) yields a more reasonable plastic indentation stress-strain response. More recently, these protocols were also shown to provide reliable estimations of uniaxial mechanical responses [20,23] and anisotropic elastic constants for cubic crystals [24].

The main goal of this work is to evaluate and demonstrate the feasibility of mechanically characterizing the material response of complex alloys such as Ti-64 at different length scales, in a systematic, comprehensive, and high throughput manner. This requires doing indentation tests from very small to very large indenter tips, loads, and displacements. Practically this involves using nanoindenters and microindenters, as shown schematically in Fig. 1. The titanium alloy Ti$6 \mathrm{Al}-4 \mathrm{~V}$ is the most common Ti alloy [2] and is a good material for such a study. In the $\beta$ annealed condition, it has a distribution of $\alpha$ and $\beta$ lamellae organized into colonies with uniform crystal orientations that are then organized into the microstructure of the material (see Fig. 1). 
The protocols of Kalidindi and Pathak [18] are employed in this work, and recent work using these protocols to study the responses of individual grains in titanium [25] as well as microindentation stress-strain curves of polycrystalline volumes in Al-6061 [23] will be leveraged to achieve our goal.

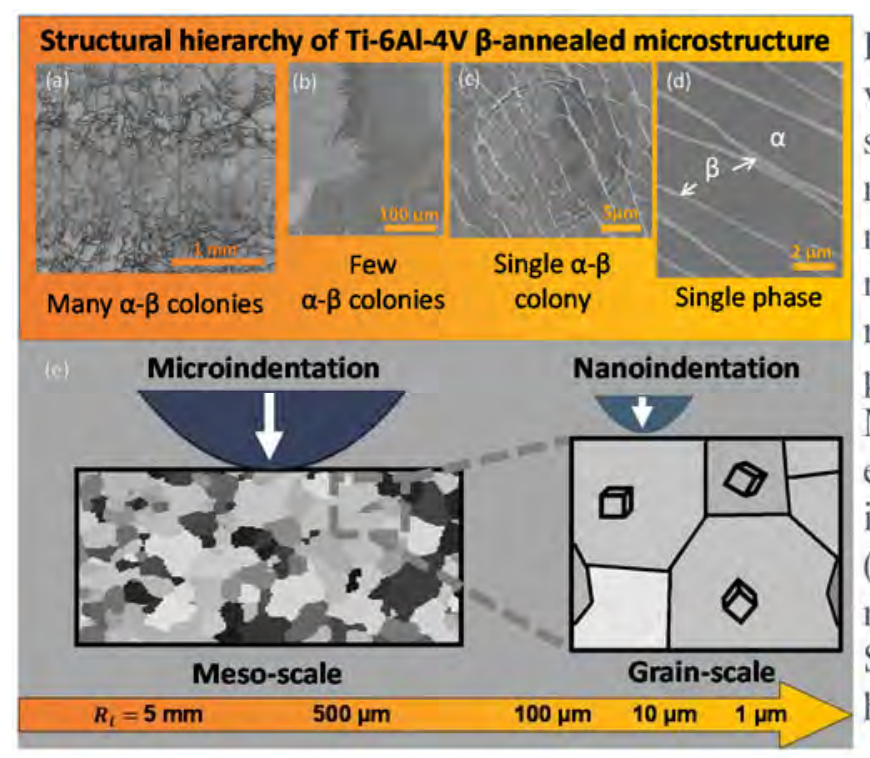

Fig. 1: (a-d) Schematic illustration of the various structural features at different length scales in Ti-64 ( $\beta$-annealed processed microstructure) and (e) indentation used for mechanical characterization. Single phase to multi-phase, polycrystalline volumes representative of the bulk material can be probed by changing the indenter size. Micrographs going from left to right are (a) electron backscatter diffraction (EBSD) image quality map showing many colonies, (b) SEM-BSE image, (c) SEM-BSE image of residual indent in a single colony, and (d) SEM-BSE image showing the two phases hcp- $\alpha$ (dark gray) and bec- $\beta$ (light gray).

\section{Materials and methods}

\subsection{Experiments}

Ti-6Al-4V in rod form was provided by Boeing in the $\beta$-annealed processed condition. The main step in the process is a slow cool down from above the $\beta$-transition temperature, which results in large $\alpha-\beta$ colonies. A single colony has uniform $\alpha$ and $\beta$ crystal orientations with alternating $\alpha$ and $\beta$ lamella. We found similar results to those reported by Smith et al. [9], which includes a strong basal texture along the axis of the rod, an average $\alpha$-lamellae thickness of 3 $\mu \mathrm{m}$, a mean colony size of $165 \mu \mathrm{m}$, and an $\alpha$-phase volume fraction of $98 \%$. Samples were sectioned and mechanically polished. A final polishing step using $0.06 \mu \mathrm{m}$ colloidal silica with hydrogen peroxide ( 5 parts colloidal silica suspension to 1 part hydrogen peroxide) was used to get a flat surface at phase and grain boundaries while removing any damaged layer from 
mechanical polishing. Electropolishing is typically used for spherical nanoindentation stressstrain measurements because it allows for material removal without any further mechanical damage [26]; however, the process sufficiently etched the $\beta$-phase so that reliable measurements could not be made (i.e., results were affected by the surface roughness). On electropolished samples, the indentation stress-strain response showed small ridges in the elastic portion, which appeared to correspond to when the contact area crossed over phase boundaries.

The relatively small $\alpha$-lamellae thickness in the $\beta$-annealed sample make it challenging to isolate the mechanical response of the $\alpha$-phase. Therefore, a second Ti-6Al-4V sample, grade 5 from McMaster-Carr, was annealed at $1025^{\circ} \mathrm{C}$ for 2 hours, slowly cooled $\left(0.1{ }^{\circ} \mathrm{C} / \mathrm{min}\right)$ to $950{ }^{\circ} \mathrm{C}$ where it was held for 1 hour, and then furnace cooled. This heat treatment, $\alpha-\beta$ anneal, produced larger uniform regions of the $\alpha$-phase to allow for reliable indentation measurements. We reported on the structure and nanoindentation stress-strain response of this material in a recent study [25]. In this prior study, electropolishing was used for preparing the samples. Here, the same sample is used but prepared following the chemo-mechanical polishing procedure described above, so that this polishing procedure can be critically evaluated. In addition, the $\alpha-$ phase response (from the $\alpha-\beta$ annealed sample) is important to this study, as it allows comparisons of the indentation stress-strain response of Ti-64 starting from the individual constituents (i.e., the $\alpha$-phase). The relatively small amount and even smaller size of $\beta$-Ti in both samples make it very difficult to isolate the mechanical response, so it was not measured.

Nanoindentation was performed on an Agilent G200 with continuous stiffness measurement (CSM). CSM allows for the contact radius to be readily determined throughout the indentation test without doing full or partial unloads [27-31]. Two conical-spherical diamond tips with a nominal radii of 16.5 and $100 \mu \mathrm{m}$ were used to probe the $\alpha$-phase response and $\alpha-\beta$ single 
colony response, respectively. Spherical tips, as opposed to sharp tip geometries (e.g., Berkovich), allow for the initial elastic response and the elastic-plastic transition to be more easily captured. This is because plasticity begins at very low loads and displacements with sharp tips compared to spherical tips. Tests were performed with a constant strain rate (loading rate divided by the load) of $0.05 / \mathrm{s}$ to displacements of approximately $450 \mathrm{~nm}$ ( $16 \mu$ m radius indenter) and $800 \mathrm{~nm}(100 \mu \mathrm{m}$ radius indenter). In many cases, the $100 \mu \mathrm{m}$ radius indenter tests hit the load limit $(\sim 700 \mathrm{mN})$ of the XP head on the nanoindenter. The CSM was used with a frequency of $45 \mathrm{~Hz}$ and a displacement amplitude of $2 \mathrm{~nm}$.

Microindentation was performed on a Zwick-Roell Z2.5 Zwicki Hardness Tester. This machine offers a much higher loading capacity $(2.5 \mathrm{kN})$; however, there is no CSM available. This requires sequential unloads in order to recover the contact radius at different stages of the test. This was achieved by doing partial (50\% of the load) unloads at a constant load increment. Spherical, tungsten-carbide, tips with nominal radii of 0.5 and $6.35 \mathrm{~mm}$ were used. The procedure for both indenter sizes was the same; however, the parameters were adjusted so that ample points on the indentation stress-strain curve were collected. A constant loading rate of 0.3 $\mathrm{mm} / \mathrm{min}$, unloading at every $40 \mathrm{~N}$, and a total of 20 unloads was used for the $6.35 \mathrm{~mm}$ indenter. A constant loading rate of $0.1 \mathrm{~mm} / \mathrm{min}$, unloading at every $2 \mathrm{~N}$, and a total of 20 unloads was used for the $0.5 \mathrm{~mm}$ indenter. The first unload in tests with the $0.5 \mathrm{~mm}$ indenter was done at $3 \mathrm{~N}$ to improve the reliability of this data point. It proved difficult to estimate the contact radius from unloads at low loads on this equipment, and in some cases, the second unload (at $5 \mathrm{~N}$ ) was taken as the first post-elastic point on the indentation stress-strain curve.

Electron microscopy was performed on a Tescan Mira XMH field emission scanning electron microscope (SEM) equipped with an EDAX Hikari camera for electron backscatter 
diffraction (EBSD). Backscattered electron (BSE) micrographs were used to capture the $\alpha-\beta$ structure at indentation sites with the $100 \mu \mathrm{m}$ radius indenter. This was done to observe clearly the area of the residual indent with respect to the $\alpha-\beta$ lamella. EBSD was used to measure the $\alpha$ phase crystal orientation in both Ti-64 samples before placing nanoindents inside single $\alpha$-grains and single $\alpha-\beta$ colonies (regions of uniform orientation). Optical images of microindentation residual indents were recorded using the camera and lenses available on the microindentation tester.

\subsection{Indentation stress-strain analysis}

The nanoindentation stress-strain protocols are well described in prior work [18, 32, 33]. These protocols were extended and utilized for microindentation testing in a recent study [23]. Also highly relevant are two recent studies using nanoindentation stress-strain protocols on hep metals $[25,34]$. Here, we will briefly review the definitions of contact radius, indentation stress, and indentation strain used, which are heavily rooted in Hertz's theory [21, 22]. Hertz's theory, Eqns. (1-4), describes the elastic, frictionless contact between two isotropic, homogenous bodies with quadratic, non-conforming surfaces,

$$
\begin{aligned}
& P=\frac{4}{3} E_{e f f} R_{e f f}^{1 / 2} h_{e}^{3 / 2} \\
& a=\sqrt{R_{e f f} h_{e}}
\end{aligned}
$$

$$
\begin{aligned}
& \frac{1}{E_{e f f}}=\frac{1-v_{i}^{2}}{E_{i}}+\frac{1-v_{S}^{2}}{E_{S}} \\
& \frac{1}{R_{e f f}}=\frac{1}{R_{i}}+\frac{1}{R_{S}}
\end{aligned}
$$

where the load, $P$, and elastic displacement, $h_{e}$, are related by the effective radius of the sampleindenter system, $R_{e f f}$, and its effective elastic modulus, $E_{e f f}$. The subscripts $i$ and $s$ denote the indenter and sample, respectively. The contact radius, $a$, is determined from experiments using the unloading stiffness, $S=d P / d h$, from CSM (nanoindentation) or direct, partial, unloads (microindentation), as

$$
a=\frac{S}{2 E_{e f f}}
$$


Definitions of indentation stress, $\sigma_{i n d}$, and indentation strain, $\varepsilon_{i n d}$, used in this study are summarized as [18-20],

$$
\begin{array}{rll}
\sigma_{\text {ind }}=\frac{P}{\pi a^{2}} & \text { (6) } & h_{s}=h-h_{i} \\
\varepsilon_{\text {ind }}=\frac{4}{3 \pi} \frac{h_{s}}{a} & \text { (7) } & h_{i}=\frac{3\left(1-v_{i}^{2}\right) P}{4 E_{i} a}
\end{array}
$$

Equations (8) and (9) are needed in order to account for the elastic displacement of the indenter tip, $h_{i}$. The correction is the theoretical equivalent to indenting the tip into a rigid flat surface, similar to how one might determine the compliance of any mechanical testing setup. Note that this is not the frame stiffness of the indenter since that is already accounted for in the data collection. The correction is very small for low loads and when the indenter modulus is much higher than the sample modulus (e.g., diamond indenting titanium). In such cases, using the total displacement, $h$, is reasonable. However, spherical microindenter tips are typically made of tungsten carbide (Young's modulus of $640 \mathrm{GPa}$ [35]) which is almost half the stiffness of diamond (Young's modulus of $1140 \mathrm{GPa}[31,36]$ ), and these tests go to very high loads. In order to ensure that the indentation stress-strain response is independent of the indenter's elastic properties, Eqns. (8) and (9) were used for both nanoindentation and microindentation.

The protocols described above have been extensively validated in prior work with different indenter sizes on a broad range of material systems [23, 25, 32, 34]. The validation has included comparisons with properties measured using alternate protocols, extensive imaging of the indenter shape, and comparisons between measurements and predictions from finite element simulations. Indeed, the protocols themselves (e.g., finding the effective zero-point, the use of a new definition of indentation strain) have been refined using the results of these studies. 
Representative indentation tests and stress-strain curves for nanoindentation and microindentation are shown in Fig. 2. There are clear differences in the load-displacement curve since microindentation tests require partial unloading. This also is the reason for the difference in the appearance of the stress-strain curves. Since the unloading stiffness is required to determine the contact radius after plastic deformation occurs, the microindentation stress-strain curve has fewer and discrete points. Each unload produces one point on the microindentation stress-strain curve. In contrast, the CSM is used in nanoindentation experiments and provides the unloading stiffness continuously so that every data point on the load-displacement curve can be converted to indentation stress and strain. Despite the differences in equipment and test protocols, the consistent use of analysis protocols allows us to compare the indentation stress-strain response across multiple lengths scales using a wide range of indenter tip sizes. Indentation stress-strain properties such as the modulus $\left(E_{\text {ind }}\right)$, yield strength $\left(Y_{\text {ind }}\right)$, and work hardening $\left(K_{\text {ind }}\right)$ can be defined. The indentation modulus comes from the initial elastic load-displacement response. Hertz's equations (specifically, Eqn. (1) and (3)) have been shown valid for elastically anisotropic materials [24, 36-41]. Eqn. (10) is a generalized form of Eqn. (3) for anisotropic elastic materials, where $E_{\text {ind }}$ is interpreted as an effective indentation modulus.

$$
\frac{1}{E_{e f f}}=\frac{1}{E_{\text {ind }}}+\frac{1-v_{i}^{2}}{E_{i}}
$$

The indentation yield strength is defined using a $0.2 \%$ strain offset on the indentation stressstrain curve $[20,23,25,34]$. The indentation work hardening in this work is determined from a linear regression of the post yield indentation stress-strain data not exceeding a strain offset of $2.5 \%$. The tests in this study were limited to moderate indentation strains. The definition of indentation work hardening from experiments, specifically how and where to determine the rate, has not been fully explored. It is expected that the rate will depend on the range of post-yield 
data used. However, the trends in the hardening rate are expected to be insensitive to the range as long as a consistent protocol is employed.

\section{Results}

The main goal of this study is to characterize and understand the indentation stress-strain response across different structural length scales in Ti-64. These structural length scales are single phase $\alpha-$ Ti-64, single colony $\alpha-\beta$ Ti-64, few colonies, and many colonies as was illustrated in Fig. 1. Indentation testing at these length scales was achieved with indenter tip radii of 16.5, 100,500 , and 6,350 $\mu \mathrm{m}$ across two different indentation machines (nano and microindenters). The nanoindentation response of $\alpha$-Ti-64 and single colony $\alpha-\beta$ Ti-64 is presented first followed by the microindentation response of few and many colonies. The trends across length scales in the indentation properties (modulus, strength, and work hardening) are then presented.

\subsection{Nanoindentation of $\alpha$-Ti-64 and single colony $\alpha-\beta$ Ti-64}

Fig. 3 shows representative indentation stress-strain curves for $\alpha$-Ti-64 (16 $\mu$ m radius indenter) and single colony $\alpha-\beta$ Ti-64 (100 $\mu \mathrm{m}$ radius indenter). As described in Section 2 , two different samples were produced and used, so that the larger $\alpha$-grains could be studied separately. Both samples underwent similar annealing heat treatments and have the same nominal chemical compositions so that the indentation responses are not affected by prior processing or chemical composition. The EBSD maps are also shown in Fig. 3 for both samples with representative indentation sites labeled. As was shown in recent work, indents along the c-axis of the $\alpha$-grain (near (0001) direction) have a much harder indentation stress-strain response than indents perpendicular to the c-axis (near (10̄10) direction) due to the differences of slip resistances and their orientation with respect to the indentation axis [25]. For example, the pyramidal $\vec{c}+\vec{a}$ slip system is the most difficult slip system and predominantly required for indents along the c-axis, 
while the prismatic $\vec{a}$ slip system is the easiest slip system and is activated in indents perpendicular to the c-axis. A similar orientation dependent response is observed in the $\alpha-\beta$ single colonies. The primary difference between the tests is the presence of small $\beta$ lamellae at the indentation site. This is evident in the BSE micrographs of the residual indents in Fig. 3. The residual indent for the single colony test near the (0001) direction is barely visible due to the shallow depth and corresponding small indentation strain $(\sim 2 \%)$. The machine load limit was reached for these tests and deeper indents could not be performed. This is also why the single colony tests (100 $\mu \mathrm{m}$ radius indenter) go to lower indentation strains than the $\alpha$-grain tests (16 $\mu \mathrm{m}$ radius indenter). The single colony residual indent for the test near the (1010) direction shows more distinct deformation because of the lower indentation stress (and slightly larger indentation plastic strain) achieved compared to the indent along the (0001) direction. There are also clear slip lines on the surface at the edges of the indent, which are aligned with prismatic slip planes. This observation is consistent with observations reported in indents in commercially pure $\alpha$-Ti titanium [25]. The average contact diameter for single colony indents at the indentation yield point is also sufficiently large, approximately $14 \mu \mathrm{m}$, to incorporate both phases and multiple lamellae. Therefore, we consider the tests with the $100 \mu \mathrm{m}$ radius indenter to represent the single colony response.

The similar response of $\alpha-\mathrm{Ti}-64$ and $\alpha-\beta$ Ti-64 colonies is not entirely surprising given the very small volume fraction of $\beta-\operatorname{Ti}(\sim 2 \%)$. In addition, the $\alpha$-orientation of the single colony is also adequate to describe the "orientation" of the colony since there is a well know orientation relationship between the $\alpha$ and $\beta$ crystals [42-44]. Fig. 4 shows the orientation trends for the indentation modulus and yield strength. The indentation properties of hcp crystals primarily depend on the declination angle of the c-axis (also the Bunge-Euler angle, $\Phi$ ) [25, 34, 45-48]. In 
Fig. 4, there are three different sets of data defined by the indenter size and final polishing procedure. The first comparison and observation to make is that the indentation properties don't depend on the polishing procedure. Indents of $\alpha$-Ti-64 for electropolished [25] and chemomechanically polished samples show good agreement; therefore, the chemo-mechanical polishing procedure is sufficient for nanoindentation stress-strain measurements. In prior work, it has been shown that the indentation stress-strain response is highly sensitive to sample preparation techniques [26], and so this is not a trivial point.

The second comparison to make is between the $\alpha-$ Ti- 64 and $\alpha-\beta$ Ti- 64 colonies. The indentation modulus of $\alpha-\beta$ Ti-64 colonies is consistently higher than $\alpha$-Ti-64 with a very similar orientation trend, Fig. 4. The elastic anisotropy of $\alpha$-Ti for various chemical compositions has been reported both as measurements of elastic constants $[49,50]$ and indentation studies $[25,45$, 48], all of which show the stiffest direction to be along the c-axis and most compliant direction being perpendicular to the c-axis. Kim and Rohklin have reported the most comprehensive study, where they measured and calculated the elastic constants using acoustic line focus microscopy on single crystal $\alpha$-Ti-6Al (Ti-6wt.\%Al) and a single colony of $\alpha-\beta$ Ti-6242 (Ti-6wt.\%Al$2 \mathrm{wt} . \% \mathrm{Sn}-4 \mathrm{wt} \% \mathrm{Zr}-2 \mathrm{Mo}$ ) [50]. The $\alpha$-phase indentation modulus and FEM calculated indentation modulus [25] of $\alpha$-Ti6Al (using their elastic constants) are in good agreement with the results presented here. Note that the $\alpha$-phase in Ti-64 is has a similar Al content to Ti-6Al with only a small amount of $\mathrm{V}[51,52]$. There is, however, a difference in the colony responses compared to the single phase responses. The reported elastic anisotropy of the $\alpha$-Ti- 6242 and colony $\alpha-\beta$ Ti6242 [50] would indicate that the indentation modulus of the colony phase is higher along the caxis $\left(\Phi=0^{\circ}\right)$ and lower for fully declined orientations $\left(\Phi=90^{\circ}\right)$ compared to the $\alpha$-phase. This is not the case in the indentation measurements of Ti-64 presented here, where colony $\alpha-\beta$ Ti-64 is 
always higher than the $\alpha$-phase. It could be due to the difference in chemical composition between Ti-64 and Ti-6242, or it could be due to the uncertainty in the elastic constants, particularly for $\beta$-Ti. Specifically, the inverse calculation used by Kim and Rohklin requires an initial guess of the elastic constants and is sensitive to this guess, as they have acknowledged [50]. The estimated elastic constants of the $\beta-T i-6242$ from single colony measurements are very similar to the initial guess using the elastic constants of $\beta-\operatorname{Ti}-15 \mathrm{Cr}$ [53]. It is not clear how accurate the elastic constants measured by Fisher and Dever [53] are for $\beta$-Ti-64 or $\beta$-Ti-6242, and a different initial estimate may lead to a different trend. Therefore, we believe that the indentation results of single colony $\alpha-\beta$ Ti-64 reported here are quite reliable, supported by the high consistency seen in the reported results in Fig. 4. A better estimate of the elastic anisotropy of $\beta$-Ti and its dependence on chemical composition is needed to fully resolve this issue.

Fig. 4 also shows a comparison of the variation of the indentation yield strength with orientation for colonies compared to the $\alpha$-grain. The measurements of $\alpha$-Ti-64 and single colony $\alpha-\beta$ Ti-64 were made with two different indenter sizes. Size effects on nanoindentation hardness measurements have been observed and discussed in literature [54-57]. However, the protocols used here to recover an indentation yield strength are largely insensitive to indenter size, particularly for well annealed materials $[25,41,58-60]$. The indentation yield strength of colony indents is marginally lower than $\alpha$-grain indents (Fig. 4). Initially this is surprising since the many $\alpha-\beta$ interfaces are expected to strengthen the material compared to $\alpha$-grains. However, the contrast in properties between $\alpha$ and $\beta$ could also cause plasticity to occur first in one phase (likely the $\alpha$-phase), specifically at phase boundaries where it is reasonable to assume there are higher stresses due to the requirement of strain compatibility and contrast in properties. This would explain why there is no increase in strength and possibly even a small drop in strength. 
The low volume fractions of the $\beta$-phase may explain why the orientation dependence remains largely unchanged between the $\alpha$-grains and the colonies.

There have been a handful of studies that measured the uniaxial mechanical response of Ti alloy single colonies $[43,44,61]$. It should be obvious that these studies were considerably more labor intensive than the spherical nanoindentation tests reported here; however, the analysis was more straight forward (i.e., slip resistances could be readily determined). These studies observed plastic anisotropy associated with the $\alpha-\beta$ interface. Namely, they observed that basal and prismatic $\vec{a}$ slip systems showed easier slip when the slip direction was closely aligned with a slip plane in $\beta-\mathrm{Ti}[43,44,61]$. As was noted earlier, the primary orientation dependence of strength observed in indentation measurements was related to the declination angle of the c-axis of the $\alpha$-phase. In indentation, it is not possible to generate single slip or isolate slip systems as achieved in uniaxial experiments. This is due to the heterogeneous nature of the stress and strain fields under the indenter. This is likely why the anisotropy of the $\alpha-\beta$ interface is not observed in the indentation measurements reported here.

\subsection{Microindentation of few and many colonies of Ti-64}

Microindentation testing was used to achieve the high loads required to indent with larger radii indenter tips. The load-displacement curves in Fig. 2 illustrate the difference in capability compared to nanoindentation. Representative indentation stress-strain responses of 0.5 and 6.35 $\mathrm{mm}$ radius indenters are shown in Fig. 5 on top of a representative $100 \mu \mathrm{m}$ radius indenter single colony response. The elastic and yield between all three indenter sizes are very similar. However, the flow stress is significantly higher for the largest indenter size. Structurally, the indentation zones for all three tips are very different. This is illustrated schematically in Fig. 6 where cross-sections of the microstructure and primary indentation zone are shown. The primary 
indentation zone contains most of the heterogeneous stress and strain field under the indenter.

The zone is approximated as a cylinder with a radius equal to the contact radius, $a$, and height of $\sim 2.4 a$. This is based on FEM simulations of isotropic materials. Here we use this approximation although the zone shape and size is likely going to depend on the material anisotropy and heterogeneity. The zone sizes in Fig. 6 are based on the estimated contact radius at the indentation yield point. It is clear that the $100 \mu \mathrm{m}$ indenter test is a single colony response, the $0.5 \mathrm{~mm}$ indenter test contains only a few colonies, and the $6.35 \mathrm{~mm}$ indenter test contains many colonies at yield. The microindentation tests are randomly placed without any knowledge of the surface and sub-surface grains at the indentation site. It is possible that some of the $0.5 \mathrm{~mm}$ radius indents only contain a single colony; however, it is more likely that the zone contains at least one colony-colony boundary. Having only a few colony boundaries in the indentation zone doesn't seem to have a significant effect on the indentation stress-strain response. The presence of the few colony boundaries may have an effect on the indentation stress-strain response at larger indentation strains. The $6.35 \mathrm{~mm}$ radius indents will always contain many colonies. The

presence of many colony boundaries appears to increase the flow stress or early work hardening of the indentation stress-strain response. This observation is reflected in the increase in the average indentation work hardening rate in the largest indenter size compared to the other sizes summarized in Table 1 and Fig. 7.

\section{Discussion}

The focus of this discussion is to extract and understand the length scale effects of indentation modulus, strength, and hardening summarized in Fig. 7 (aggregated from all the indentation measurements obtained and discussed earlier). Each data set was collected with a different indenter size chosen specifically to probe a structural length scale in Ti-64. The 
smallest indenter size (16 $\mu \mathrm{m}$ radius) was used to characterize the $\alpha$-phase in Ti- 64 . The next size up (100 $\mu$ m radius) was used to measure the response of single $\alpha-\beta$ colonies. The primary indentation zone of the $500 \mu \mathrm{m}$ radius indenter at yield contained only a few colonies, and the $6,350 \mu \mathrm{m}$ radius indenter contained many colonies. The changes in the indentation response with indenter size directly reflect the changes in the structure/length scales in the primary indentation zone. The indentation modulus and yield strength are plotted against the contact diameter $(2 a)$ at yield, and the work hardening is plotted against the average contact diameter over the selected hardening region (described earlier).

There are two increases in the average indentation modulus. The first occurs going from $\alpha$-Ti-64 to single colony $\alpha-\beta$ Ti-64. This was shown more clearly in Fig. 4 and discussed in Section 3.1. The measurements reflect an increase in the stiffness of the colony structure compared to the $\alpha$-phase. The next increase in the indentation modulus is for microindentation tests ( 0.5 and $6.35 \mathrm{~mm}$ radius indenters). This is likely due to the strong basal texture of the material. The microindents were performed along the rod-axis in the direction of the basal texture, and they were randomly placed. The sampling and average modulus then is likely to reflect the texture of the material compared to nanoindentation experiments, which were precisely chosen to fall in a range of grain and colony orientations. Indeed, the microindentation indentation modulus does not exceed the range measured with nanoindentation, but is rather just a shift toward the stiffer c-axis indentation modulus. This is promising for spherical microindentation stress-strain protocols with tips of a sufficiently large size as a tool for characterizing the effects of texture on the mechanical response in a high throughput manner.

The average indentation yield strength, however, remains relatively constant for all indenter sizes. This seems to indicate that the $\alpha$-phase, which makes up $98 \%$ of the $\beta$-annealed 
microstructure, is controlling the indentation yield strength. This would also imply that plastic deformation begins in this phase (see Fig. 4). When each indentation test/grain average is plotted, the variance in strength is greatest for $\alpha$-phase indents. This makes sense since $\alpha$-Ti is known to exhibit significant plastic anisotropy $[8,62-66]$. The variance in strength is significantly reduced when the effect of single phase and single colonies is homogenized under the largest indenter size, which creates a large primary indentation zone of many colonies. These finding would also seem to support the fact that indentation yield strength measurements in annealed metals don't show significant size effects [25, 41, 58-60]. In other words, the average of multiple nanoindentation measurements made on randomly selected single grains or phases reasonably represents the bulk indentation strength (i.e., the bulk strength is a homogenized response of the constituents). Recent work has shown that the indentation yield strength using the protocols followed in this work is approximately 2 times higher than the uniaxial yield strength for isotropic materials [20,23]. The average indentation strength of the largest indenter size is approximately 1.9 times the reported tensile strength of $955 \mathrm{MPa}$ for the same material [9], which is remarkably close given that the ratio is likely to be affected by the material anisotropy. The entire indentation stress-strain response can also be scaled to a uniaxial true stress-strain response [20] using data available from literature [67], Fig. 8. Given potential differences between our samples and those reported in literature, and the uncertainty regarding the degree of anisotropy exhibited by the samples, the results are in good agreement. This strength data provides a unique set of observations for microstructural models (e.g., crystal plasticity models). The response at different structural length scales can be used to calibrate multiscale model parameters and/or improve the physics of the multiscale models so that they are 
well matched to the high throughput, multiscale observations made here with the novel spherical indentation stress-strain protocols described here.

The indentation hardening shows probably the most interesting trend, in that it increases significantly for the largest indenter size. The determination of indentation work hardening rates from indentation stress-strain experiments has not been sufficiently explored, so caution should be used. The exact values of the indentation hardening rate are likely dependent on the range over which the hardening rate is calculated. The end of the range for the hardening fit is listed in Table 1. Only moderate indentation strains were achieved in the tests in these studies. If we take the exact indentation hardening rates calculated from yield to moderate strains, the values are 0.7-1.4 times the shear modulus ( $44 \mathrm{GPa}$ ). Conventionally, this is rather high since most bulk uniaxial hardening rates are a fraction of the shear modulus [68].

One reason for the increased hardening rates in larger indenters in hcp metals discussed by Pathak et al. [34] is the activation of vary hard slip modes (e.g., $\vec{c}+\vec{a}$ pyramidal slip system) and/or the propensity for twinning. However, twinning is not likely occurring in Ti-6Al-4V, and the hardening rates to shear modulus ratio exceeds even the highest values reported by Pathak et al. [34]. Rather the high values of hardening rates obtained in this study are likely in part due to the strain range over which the hardening rate was calculated. One of the factors making it difficult to extract the hardening models from the indentation experiments is the large elasticplastic transition seen in the indentations stress-strain curves [19,20]. As a result, it is likely that the strain hardening regimes selected for extracting the hardening rates are still influenced by the elastic-plastic transition (an avoidable feature in the indentation stress-strain protocols). Tests to larger depths and subsequent indentation strains are required to fully develop the extraction of meaningful hardening rates. On the other hand, the trend in hardening rate is not likely to change 
and reflects the higher increase in flow stress after yield at moderate strains for the largest indenter size as was shown in Fig. 5.

The presence of many colony boundaries in the largest indenter size is likely driving the increase in flow stress and higher indentation hardening rate. In a much simpler system, Hou et al. [69] varied the grain size of copper samples while keeping the indenter size fixed. When the projected contact area became 6 times or larger than grain size, a Hall-Petch like relationship between hardness and grain size was observed. It is difficult to compare the work by Hou et al. [69] with the present work due to the difference in protocols; however, it appears that they are in agreement. When a sufficient number of interfaces (in this study, colony boundaries) are present in the indentation zone, the flow stress will increase compared to single grain measurements. Note that the hardness in the Hou et al. study [69] is more appropriately related to the flow stress in this study, since it is an indentation stress well after the indentation yield point. The role of grain and phase boundaries across a range of materials is not yet fully understood. However, the novel experiments and protocols presented here have demonstrated clearly the ability to generate a reliable data set that can be used to further understand the roles of phase and grain boundaries in deformation in a highly systematic and high throughput manner.

\section{Conclusions}

The main conclusions from this study are as follows:

1. Spherical indentation (nano and microindentation) stress-strain measurements can be used to mechanically characterize the structural hierarchy in complex Ti alloys in a high throughput manner. This includes single phase, multi-phase, and polycrystalline structures containing various numbers of grains. This is important for rapidly discovering 
new materials and new physical phenomenon through the interaction of modeling and experiments.

2. Indentation yield strength measurements do not show significant size effects from nano to microindentation in annealed metals. This is supported by the observation that largest indenter size results (many colonies) are equivalent to an averaging of the single colony measurements at lower length scales. The indentation modulus shows the same behavior which also reflects the texture of the material at higher length scales.

3. The presence of $\alpha-\beta$ boundaries or few colony boundaries in the primary indentation zone does not increase the indentation flow stress at moderate plastic strains; rather, a critical amount of colony boundaries is required before the indentation response will work harden at a higher rate. This indicates that the colony-colony boundaries are critical for understanding the mechanical behavior of this alloy.

\section{Acknowledgements}

The authors gratefully acknowledge funding by the National Science Foundation (NSF CMMI1333083). Any opinions, findings, and conclusions or recommendations expressed here are those of the authors and do not necessarily reflect the views of the NSF. We also wish to thank Dr. Don Shih of Boeing for his support and insight in addition to providing material for this study. We are grateful for the assistance of Mr. Dipen Patel in calculating the orientation dependent regression fits using spherical harmonics. Lastly, the authors wish to acknowledge the many helpful discussions with Mr. Matthew W. Priddy and Dr. David L. McDowell and their astute comments.

\section{References}

1. Donachie, M.J., Titanium : a technical guide. 2nd ed. 2000, Materials Park, OH: ASM International. vii, $381 \mathrm{p}$. 
2. Lütjering, G. and J.C. Williams, Titanium. 2nd ed. Engineering materials and processes,. 2007, Berlin ; New York: Springer. xii, $442 \mathrm{p}$.

3. Zhao, A.M., et al., The flow behavior and microstructure evolution during $(\alpha+\beta)$ deformation of B wrought TA15 titanium alloy. Materials \& Design, 2016. 109: p. 112-122.

4. Qiu, C., et al., A new approach to develop palladium-modified Ti-based alloys for biomedical applications. Materials \& Design, 2016. 109: p. 98-111.

5. Vrancken, B., et al., Microstructure and mechanical properties of a novel 8 titanium metallic composite by selective laser melting. Acta Materialia, 2014. 68: p. 150-158.

6. Liu, L.H., et al., A new insight into high-strength Ti62Nb12.2Fe13.6Co6.4Al5.8 alloys with bimodal microstructure fabricated by semi-solid sintering. Scientific Reports, 2016. 6: p. 23467.

7. Fan, J.K., et al., Characterization of hot deformation behavior of a new near beta titanium alloy: Ti-7333. Materials \& Design, 2013. 49: p. 945-952.

8. Mayeur, J.R. and D.L. McDowell, A three-dimensional crystal plasticity model for duplex Ti-6Al4V. International Journal of Plasticity, 2007. 23(9): p. 1457-1485.

9. Smith, B.D., D. Shih, and D.L. McDowell, Cyclic Plasticity Experiments and Polycrystal Plasticity Modeling of Three Distinct Ti Alloy Microstructures. International Journal of Plasticity, 2013.

10. Wu, G.Q., et al., Effect of microstructure on the fatigue properties of Ti-6Al-4V titanium alloys. Materials \& Design, 2013. 46: p. 668-674.

11. Katani, S., et al., Micromechanical modelling of damage behaviour of Ti-6Al-4V. Materials \& Design, 2013. 49: p. 1016-1021.

12. Ji, Z., H. Yang, and H.W. Li, Predicting the effects of microstructural features on strain localization of a two-phase titanium alloy. Materials \& Design, 2015. 87: p. 171-180.

13. Tabor, D., The hardness of metals. 1951, Oxford,: Clarendon Press. ix, 175 p.

14. Field, J.S. and M.V. Swain, A Simple Predictive Model for Spherical Indentation. Journal of Materials Research, 1993. 8(2): p. 297-306.

15. Field, J.S. and M.V. Swain, Determining the mechanical properties of small volumes of material from submicrometer spherical indentations. Journal of Materials Research, 1995. 10(1): p. 101112.

16. Herbert, E.G., et al., On the measurement of stress-strain curves by spherical indentation. Thin Solid Films, 2001. 398: p. 331-335.

17. Basu, S., A. Moseson, and M.W. Barsoum, On the determination of spherical nanoindentation stress-strain curves. Journal of Materials Research, 2006. 21(10): p. 2628-2637.

18. Kalidindi, S.R. and S. Pathak, Determination of the effective zero-point and the extraction of spherical nanoindentation stress-strain curves. Acta Materialia, 2008. 56(14): p. 3523-3532.

19. Donohue, B.R., A. Ambrus, and S.R. Kalidindi, Critical evaluation of the indentation data analyses methods for the extraction of isotropic uniaxial mechanical properties using finite element models. Acta Materialia, 2012. 60(9): p. 3943-3952.

20. Patel, D.K. and S.R. Kalidindi, Correlation of spherical nanoindentation stress-strain curves to simple compression stress-strain curves for elastic-plastic isotropic materials using finite element models. Acta Materialia, 2016. 112: p. 295-302.

21. Hertz, H., D.E. Jones, and G.A. Schott, Miscellaneous papers. 1896, London, New York: Macmillan and co. xxvi, $340 \mathrm{p}$.

22. Johnson, K.L., Contact mechanics. 1985, Cambridge Cambridgeshire ; New York: Cambridge University Press. xi, $452 \mathrm{p}$.

23. Weaver, J.S., et al., High throughput exploration of process-property linkages in Al-6061 using instrumented spherical microindentation and microstructurally graded samples. Integrating Materials and Manufacturing Innovation, 2016. 5(1): p. 1-20. 
24. Patel, D.K., H.F. Al-Harbi, and S.R. Kalidindi, Extracting single-crystal elastic constants from polycrystalline samples using spherical nanoindentation and orientation measurements. Acta Materialia, 2014. 79: p. 108-116.

25. Weaver, J.S., et al., On capturing the grain-scale elastic and plastic anisotropy of alpha-Ti with spherical nanoindentation and electron back-scattered diffraction. Acta Materialia, 2016. 117: p. 23-34.

26. Pathak, S., et al., Importance of surface preparation on the nano-indentation stress-strain curves measured in metals. Journal of Materials Research, 2009. 24(3): p. 1142-1155.

27. J. Hay, P.A., and E. Herbert, Continuous Stiffness Measurement During Instrumented Indentation Testing. Experimental Techniques, 2010. 86.

28. Li, X.D. and B. Bhushan, A review of nanoindentation continuous stiffness measurement technique and its applications. Materials Characterization, 2002. 48(1): p. 11-36.

29. Pharr, G.M., J.H. Strader, and W.C. Oliver, Critical issues in making small-depth mechanical property measurements by nanoindentation with continuous stiffness measurement. Journal of Materials Research, 2009. 24(3): p. 653-666.

30. Vachhani, S.J., R.D. Doherty, and S.R. Kalidindi, Effect of the continuous stiffness measurement on the mechanical properties extracted using spherical nanoindentation. Acta Materialia, 2013. 61(10): p. 3744-3751.

31. Oliver, W.C. and G.M. Pharr, Measurement of hardness and elastic modulus by instrumented indentation: Advances in understanding and refinements to methodology. Journal of Materials Research, 2004. 19(1): p. 3-20.

32. Pathak, S. and S.R. Kalidindi, Spherical nanoindentation stress-strain curves. Materials Science \& Engineering R-Reports, 2015. 91: p. 1-36.

33. Pathak, S., J. Shaffer, and S.R. Kalidindi, Determination of an effective zero-point and extraction of indentation stress-strain curves without the continuous stiffness measurement signal. Scripta Materialia, 2009. 60(6): p. 439-442.

34. Pathak, S., S.R. Kalidindi, and N.A. Mara, Investigations of orientation and length scale effects on micromechanical responses in polycrystalline zirconium using spherical nanoindentation. Scripta Materialia, 2016. 113: p. 241-245.

35. ASM International, ASM handbooks online. 2002, ASM International: Materials Park, $\mathrm{OH}$.

36. Vlassak, J.J., et al., The indentation modulus of elastically anisotropic materials for indenters of arbitrary shape. Journal of the Mechanics and Physics of Solids, 2003. 51(9): p. 1701-1721.

37. Vlassak, J.J. and W.D. Nix, Measuring the elastic properties of anisotropic materials by means of indentation experiments. Journal of the Mechanics and Physics of Solids, 1994. 42(8): p. 12231245.

38. Vlassak, J.J. and W.D. Nix, Indentation Modulus of Elastically Anisotropic Half-Spaces. Philosophical Magazine a-Physics of Condensed Matter Structure Defects and Mechanical Properties, 1993. 67(5): p. 1045-1056.

39. Willis, J.R., Hertzian Contact of Anisotropic Bodies. Journal of the Mechanics and Physics of Solids, 1966. 14(3): p. 163-\&.

40. Gao, Y.F. and G.M. Pharr, Multidimensional contact moduli of elastically anisotropic solids. Scripta Materialia, 2007. 57(1): p. 13-16.

41. Pathak, S., D. Stojakovic, and S.R. Kalidindi, Measurement of the local mechanical properties in polycrystalline samples using spherical nanoindentation and orientation imaging microscopy. Acta Materialia, 2009. 57(10): p. 3020-3028.

42. Burgers, W.G., On the process of transition of the cubic-body-centered modification into the hexagonal-close-packed modification of zirconium. Physica, 1934. 1: p. 561-586. 
43. Savage, M.F., et al., Deformation mechanisms and microtensile behavior of single colony Ti6242Si. Materials Science and Engineering a-Structural Materials Properties Microstructure and Processing, 2001. 319: p. 398-403.

44. Salem, A.A. and S.L. Semiatin, Anisotropy of the hot plastic deformation of Ti-6Al-4V singlecolony samples. Materials Science and Engineering a-Structural Materials Properties Microstructure and Processing, 2009. 508(1-2): p. 114-120.

45. Britton, T.B., et al., The effect of crystal orientation on the indentation response of commercially pure titanium: experiments and simulations. Proceedings of the Royal Society a-Mathematical Physical and Engineering Sciences, 2010. 466(2115): p. 695-719.

46. Zambaldi, C., et al., Orientation informed nanoindentation of alpha-titanium: Indentation pileup in hexagonal metals deforming by prismatic slip. Journal of Materials Research, 2012. 27(1): $p$. 356-367.

47. Viswanathan, G.B., et al., Direct observations and analyses alpha phase of an alpha/beta Ti-alloy of dislocation substructures in the formed by nanoindentation. Acta Materialia, 2005. 53(19): $p$. 5101-5115.

48. Kwon, J., et al., Characterization of deformation anisotropies in an alpha-Ti alloy by nanoindentation and electron microscopy. Acta Materialia, 2013. 61(13): p. 4743-4756.

49. Simmons, G. and H. Wang, Single crystal elastic constants and calculated aggregate properties: a handbook. 2d ed. 1971, Cambridge, Mass.,: M.I.T. Press. xv, 370 p.

50. Kim, J.Y. and S.I. Rokhlin, Determination of elastic constants of generally anisotropic inclined lamellar structure using line-focus acoustic microscopy. Journal of the Acoustical Society of America, 2009. 126(6): p. 2998-3007.

51. Atapour, M., et al., Corrosion Behavior of Ti-6Al-4V with Different Thermomechanical Treatments and Microstructures. Corrosion, 2010. 66(6).

52. Atapour, M., et al., Corrosion behaviour of investment cast and friction stir processed Ti-6Al-4V. Corrosion Science, 2010. 52(9): p. 3062-3069.

53. Fisher, E.S. and D. Dever, The single crystal elastic moduli of beta-titanium and titaniumchromium alloys, in The Science, Technology and Application of Titanium, N.E. Promisel, Editor. 1970, Pergamon. p. 373-381.

54. Huang, Y., et al., A model of size effects in nano-indentation. Journal of the Mechanics and Physics of Solids, 2006. 54(8): p. 1668-1686.

55. Nix, W.D. and H.J. Gao, Indentation size effects in crystalline materials: A law for strain gradient plasticity. Journal of the Mechanics and Physics of Solids, 1998. 46(3): p. 411-425.

56. Swadener, J.G., E.P. George, and G.M. Pharr, The correlation of the indentation size effect measured with indenters of various shapes. Journal of the Mechanics and Physics of Solids, 2002. 50(4): p. 681-694.

57. Pharr, G.M., E.G. Herbert, and Y.F. Gao, The Indentation Size Effect: A Critical Examination of Experimental Observations and Mechanistic Interpretations. Annual Review of Materials Research, Vol 40, 2010. 40: p. 271-292.

58. Kalidindi, S.R. and S.J. Vachhani, Mechanical characterization of grain boundaries using nanoindentation. Current Opinion in Solid State \& Materials Science, 2014. 18(4): p. 196-204.

59. Vachhani, S.J. and S.R. Kalidindi, Grain-scale measurement of slip resistances in aluminum polycrystals using spherical nanoindentation. Acta Materialia, 2015. 90: p. 27-36.

60. Pathak, S., et al., Studying grain boundary regions in polycrystalline materials using spherical nano-indentation and orientation imaging microscopy. Journal of Materials Science, 2012. 47(2): p. 815-823. 
61. Suri, S., et al., Room temperature deformation and mechanisms of slip transmission in oriented single-colony crystals of an alpha/beta titanium alloy. Acta Materialia, 1999. 47(3): p. 10191034.

62. Williams, J.C., R.G. Baggerly, and N.E. Paton, Deformation behavior of HCP Ti-Al alloy single crystals. Metallurgical and Materials Transactions A, 2002. 33(3): p. 837-850.

63. Britton, T.B., F.P.E. Dunne, and A.J. Wilkinson, On the mechanistic basis of deformation at the microscale in hexagonal close-packed metals. Proceedings of the Royal Society a-Mathematical Physical and Engineering Sciences, 2015. 471(2178).

64. Fundenberger, J.J., et al., Modelling and prediction of mechanical properties for materials with hexagonal symmetry (zinc, titanium and zirconium alloys). Acta Materialia, 1997. 45(10): $p$. 4041-4055.

65. Sakai, T. and M.E. Fine, Basal slip of Ti-Al single crystals. Scripta Metallurgica, 1974. 8(5): p. 545547.

66. Sakai, T. and M.E. Fine, Plastic deformation of Ti-Al single crystals in prismatic slip. Acta Metallurgica, 1974. 22(11): p. 1359-1372.

67. Atlas of Stress-Strain Curves. 2 ed. 2002, Materials Park, OH: ASM International.

68. Callister, W.D., Materials science and engineering : an introduction. 4th ed. 1997, New York: John Wiley \& Sons. $x x, 852$ p., 4 p. of plates.

69. Hou, X.D., A.J. Bushby, and N.M. Jennett, Study of the interaction between the indentation size effect and Hall-Petch effect with spherical indenters on annealed polycrystalline copper. Journal of Physics D-Applied Physics, 2008. 41(7). 


\section{Figure Captions}

Fig. 1: (a-d) Schematic illustration of the various structural features at different length scales in Ti-64 ( $\beta$-annealed processed microstructure) and (e) indentation used for mechanical characterization. Single phase to multi-phase, polycrystalline volumes representative of the bulk material can be probed by changing the indenter size. Micrographs going from left to right are (a) electron backscatter diffraction (EBSD) image quality map showing many colonies, (b) SEMBSE image, (c) SEM-BSE image of residual indent in a single colony, and (d) SEM-BSE image showing the two phases hcp- $\alpha$ (dark gray) and bcc- $\beta$ (light gray).

Fig. 2: The mechanical response from spherical nano $(a, b)$ and microindentation $(c, d)$ tests. $(a, c)$ are load-displacement curves, and $(b, d)$ are indentation stress-strain curves. The nanoindentation response is for a single colony using a $100 \mu \mathrm{m}$ radius indenter, and the microindentation response is for many colonies using a $6.35 \mathrm{~mm}$ radius indenter.

Fig. 3: (a) EBSD inverse pole figure map of the $\alpha-\beta$ annealed microstructure with regions labeled that are suitable for $\alpha$-phase ( $\alpha$-Ti-64) indentation. (b) EBSD inverse pole figure of the $\beta$ annealed microstructure with regions labeled that are suitable for single colony $\alpha-\beta$ indentation.

(c) The nanoindentation stress-strain response for $\alpha$-Ti-64 and single colony $\alpha-\beta$ Ti-64 for $\alpha$ orientations near (0001), and (d) corresponding SE-BSE micrograph of the single colony residual indent. (e) The nanoindentation stress-strain response for $\alpha$-Ti-64 and single colony $(\alpha-\beta)$ Ti-64 for $\alpha$-orientations near $(10 \overline{1} 0)$, and (f) corresponding SE-BSE micrograph of the single colony residual indent. The insets in (c) and (e) show the top-down view (view from the indenter) of the $\alpha$-crystal orientation of the colonies.

Fig. 4: (a-c) Inverse pole figures for the grains tested for three different scenarios (a) electropolished $\alpha$-phase indents, (b) chemo-mechanically polished $\alpha$-phase indents, and (c) chemo-mechanically polished $\alpha-\beta$ colony indents. The colonies are represented solely by their $\alpha$ orientation. (d) The indentation modulus versus the $\alpha$-crystal declination angle. (e) The indentation yield strength versus the $\alpha$-crystal declination angle. The solid lines represent a regression fit to the data sets using a spherical harmonic function.

Fig. 5: (a) Representative indentation stress-strain curves of Ti-64 $\beta$-annealed samples with different indenter sizes. Representative residual indent for (b) $500 \mu \mathrm{m}$ radius indenter tests and (c) $6,350 \mu \mathrm{m}$ radius indenter tests.

Fig. 6: Schematic representation of the primary indentation zone at the indentation yield point with respect to the structural length scales in Ti-64 $\beta$-annealed for different indenter sizes. The zone is approximated as a cylinder with a diameter of $2 \mathrm{x}$ the contact radius, $a$, and a height of $2.4 a$.

Fig. 7: The indentation stress-strain properties (modulus, strength, and work hardening) for Ti6Al-4V measured with four different indenter sizes. (a) Pictorial representation of the different structural features tested with each indenter size. (b) The average indentation modulus for each indenter size versus the contact diameter at yield. The indentation yield strength versus contact diameter at yield for (c) each grain/test average and (d) the total average. The indentation work hardening rate versus the average contact diameter over the hardening fit for (e) each grain/test average and (f) the total average. The error bars represent one standard deviation. 
Fig. 8: The scaled indentation stress-strain response from the largest indenter size (radius of 6.35 $\mathrm{mm}$ ) compared to different tensile $(\mathrm{T})$ and compression $(\mathrm{C})$ true stress-strain curves in literature [67]. The scaling factors from indentation stress-strain to uniaxial true stress-strain were taken from [20].

\section{Tables}

Table 1: Average indentation stress-strain measurements for each indenter size. The properties include the indentation modulus, $E_{\text {ind }}$, indentation yield strength, $Y_{\text {ind }}$, indentation work hardening rate, $K_{\text {ind }}$, and the contact radius, $a$, at different points during the test. The indentation yield strength and contact radius at yield were determined using a $0.2 \%$ strain offset. The hardening rate was determined using a linear fit between the yield point and the maximum strain offset. The contact radius for this fit is the average of this range. It was limited to relatively small indentation strains. The error bars are all one standard deviation.

\begin{tabular}{|c|c|c|c|c|c|c|c|c|}
\hline $\mathbf{R}_{\mathrm{i}}[\boldsymbol{\mu m}]$ & $\begin{array}{l}\text { Local } \\
\text { Structure }\end{array}$ & $\mathrm{E}_{\text {ind }}[\mathrm{GPa}]$ & $\mathbf{Y}_{\text {ind }}[\mathrm{MPa}]$ & $\begin{array}{l}\text { a@yield } \\
{[\mu \mathrm{m}]}\end{array}$ & $\mathrm{K}_{\text {ind }}$ [GPa] & $\begin{array}{l}\text { a for } K_{\text {ind }} \\
{[\mu \mathrm{m}]}\end{array}$ & $\begin{array}{l}\text { Strain offset } \\
\text { for } K_{\text {ind }}[\%]\end{array}$ & $\begin{array}{l}\text { Max Load } \\
{[\mathrm{N}]}\end{array}$ \\
\hline 16 & $\alpha-\mathrm{Ti}-64$ & $113 \pm 12.2$ & $1834 \pm 321$ & $0.79 \pm 0.06$ & $32.1 \pm 9.8$ & $1.93 \pm 0.27$ & $2.1 \pm 0.2$ & 0.09 \\
\hline 100 & $\alpha-\beta$ colony & $116 \pm 9.1$ & $1718 \pm 305$ & $3.49 \pm 0.32$ & $33.7 \pm 16.1$ & $6.43 \pm 0.37$ & $1.1 \pm 0.2$ & 0.70 \\
\hline 500 & few colony & $129 \pm 10.8$ & $1727 \pm 261$ & $16.7 \pm 2.8$ & $29.9 \pm 8.6$ & $44.7 \pm 4.4$ & $1.9 \pm 0.3$ & 41 \\
\hline 6350 & $\begin{array}{l}\text { many } \\
\text { colonies }\end{array}$ & $137 \pm 9.1$ & $1832 \pm 197$ & $219.1 \pm 15.0$ & $62.4 \pm 61$ & $273.6 \pm 12.2$ & $0.8 \pm 0.1$ & 800 \\
\hline
\end{tabular}



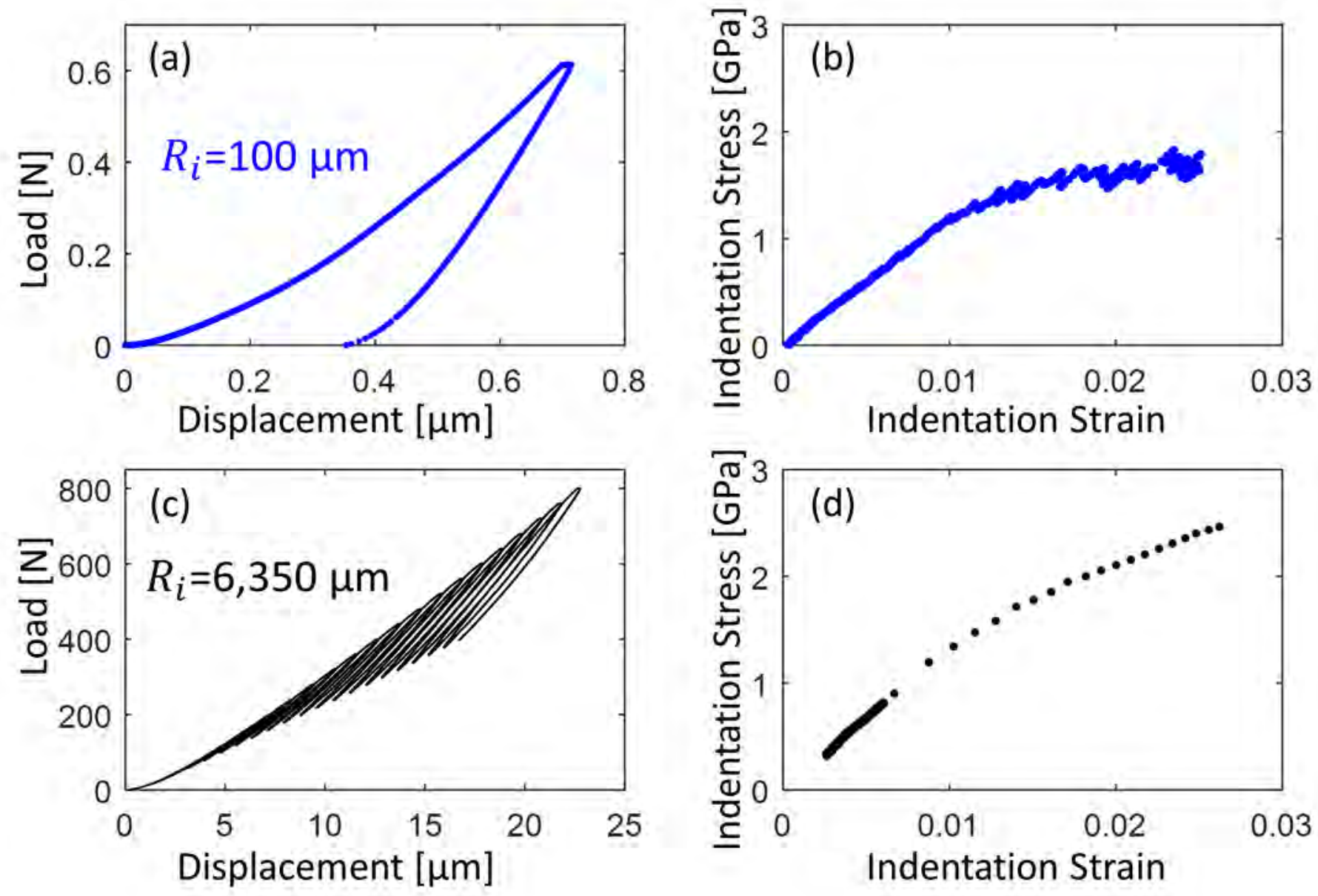

Figure 2. 

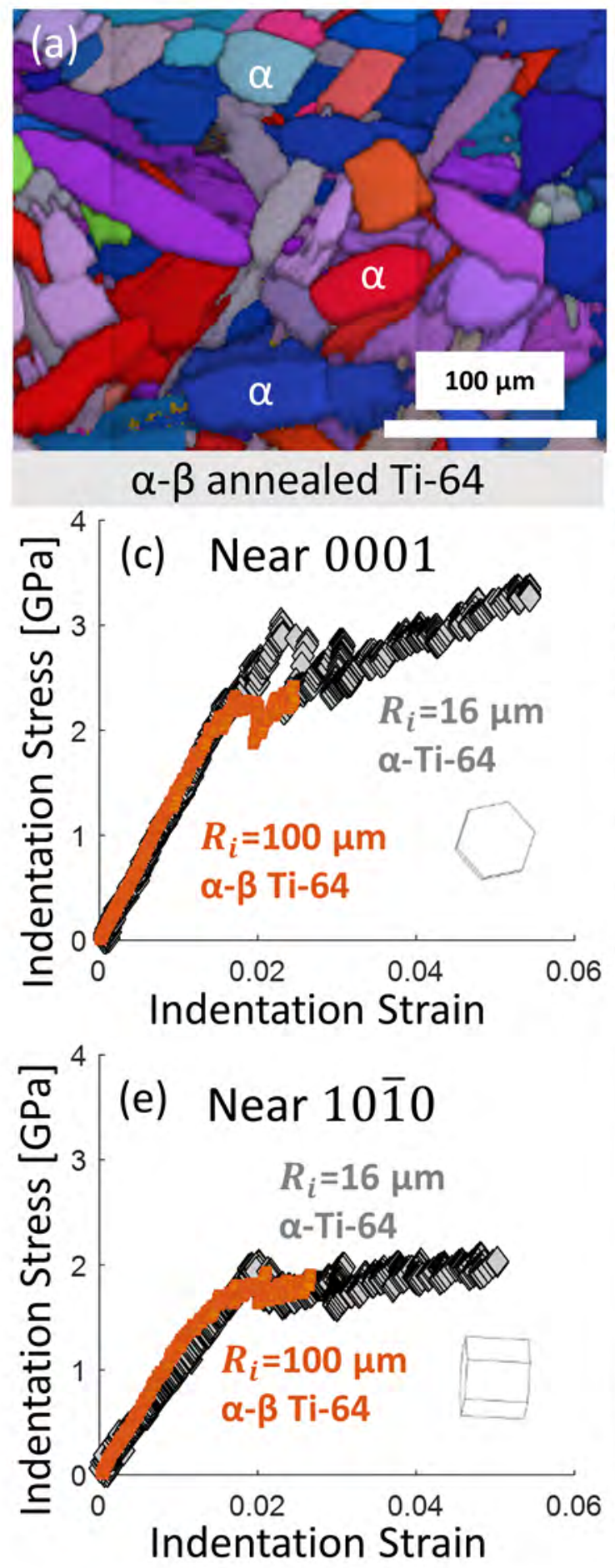

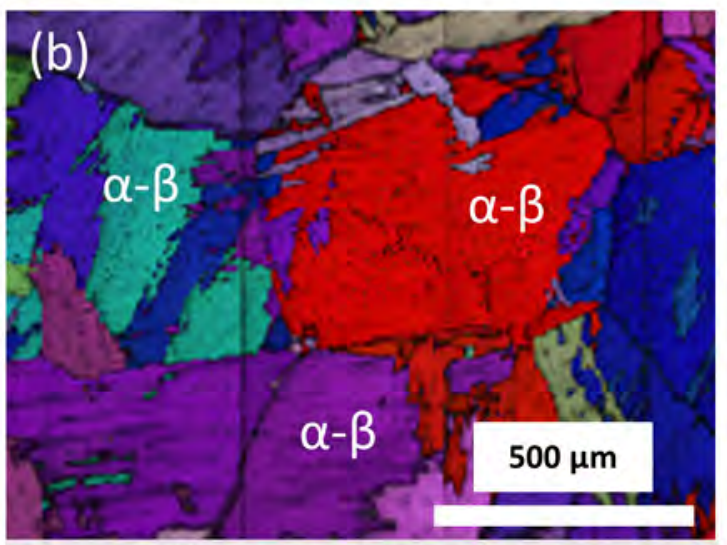

$\beta$-annealed Ti-64

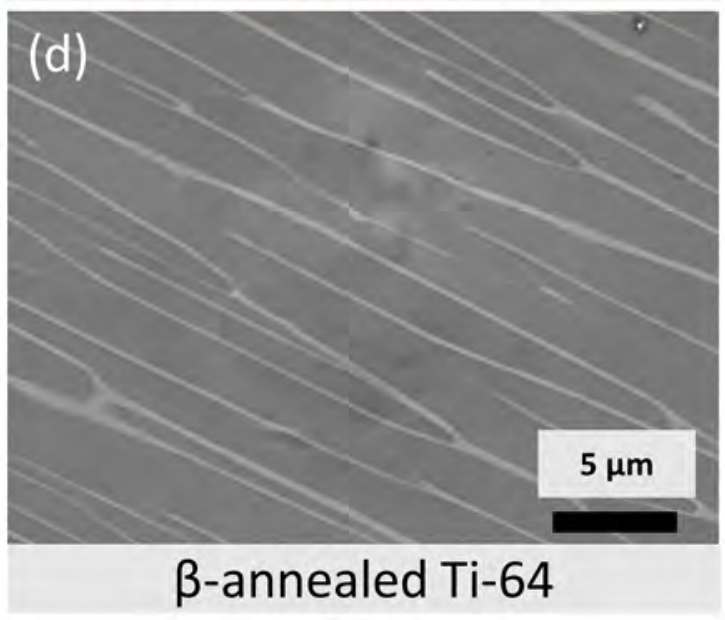

(f)

$5 \mu \mathrm{m}$

$\beta$-annealed Ti-64

Figure 3. 
(a)

electropolish $R_{i}=16 \mu \mathrm{m}$ $\alpha-T i-64$

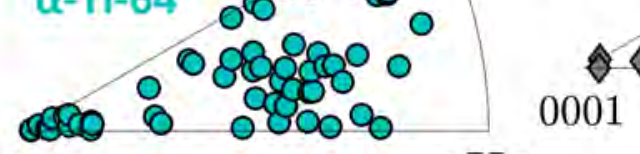

0001

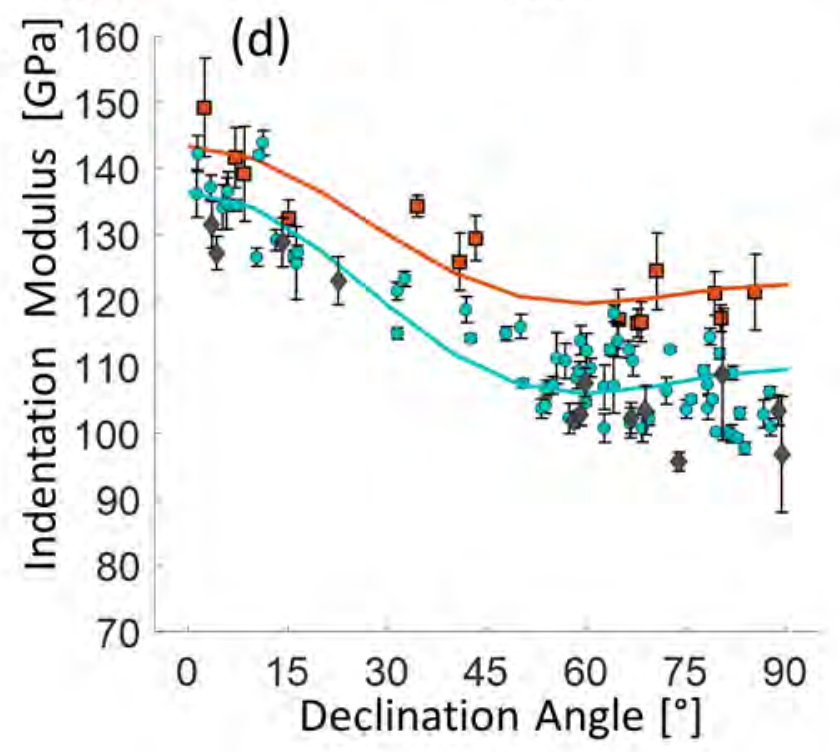
$R_{i}=16 \mu \mathrm{m}$ $\alpha-\mathrm{Ti}-64$ 888
80
80
0 $\begin{array}{lll}\text { (b) chemo-mech.polish } 10 \overline{1} 0 & \text { (c) }\end{array}$ $\diamond$

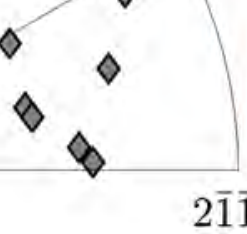

chemo-mech. polish $R_{i}=100 \mu \mathrm{m}$

$\alpha-\beta$ Ti-64

$2 \overline{1} \overline{1} 0$
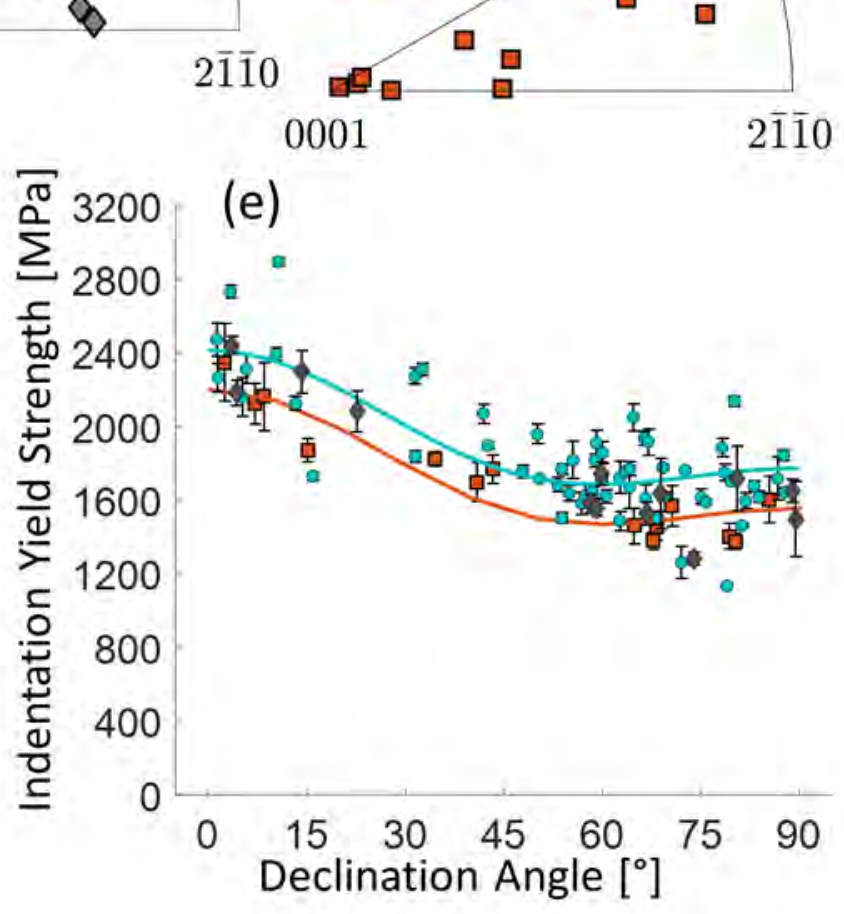

Figure 4. 

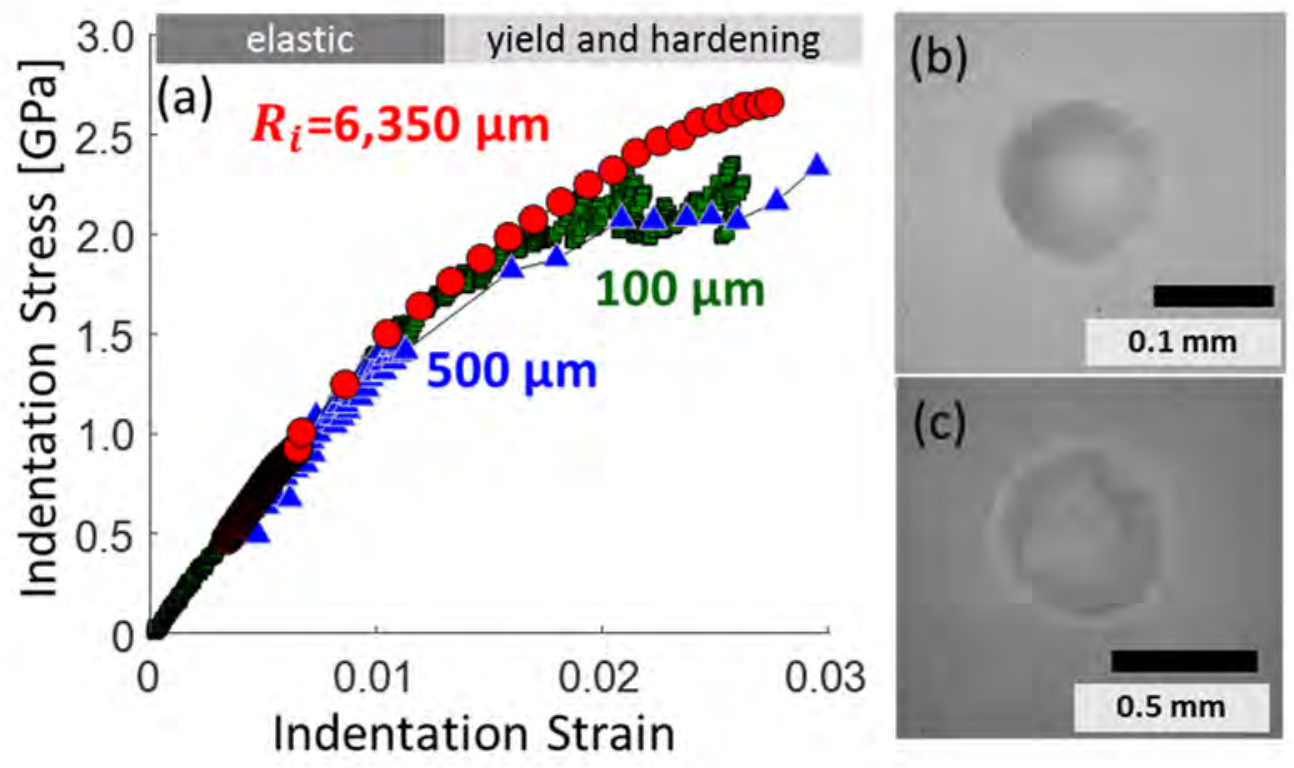

Figure 5. 


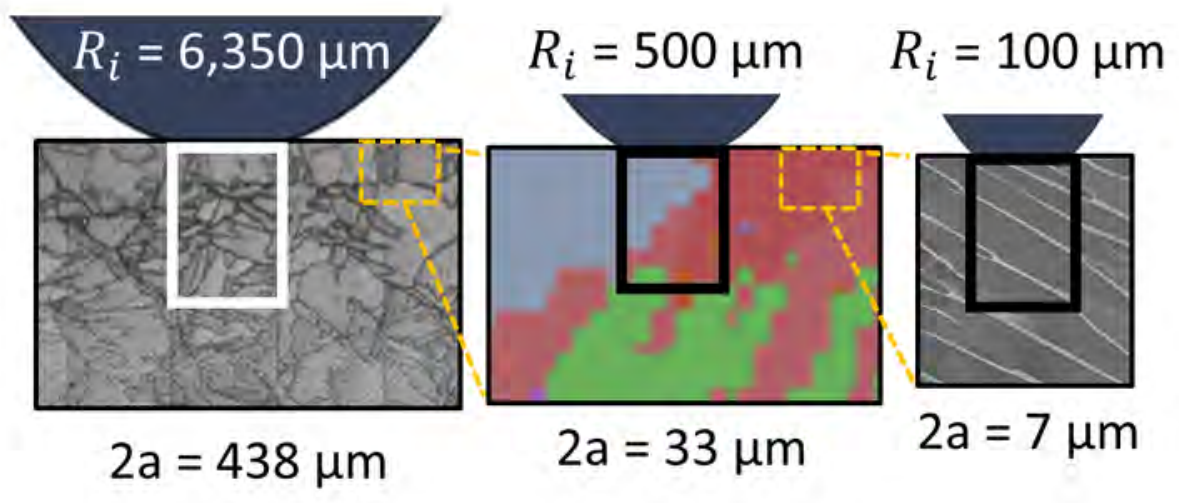

Figure 6. 
(a)

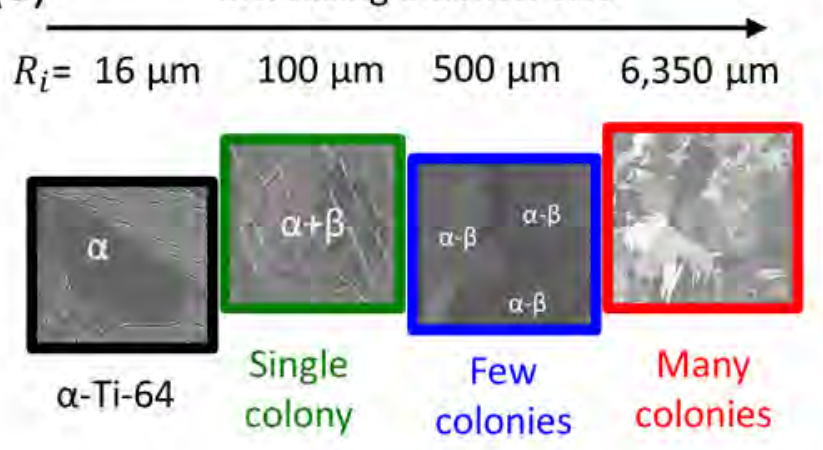

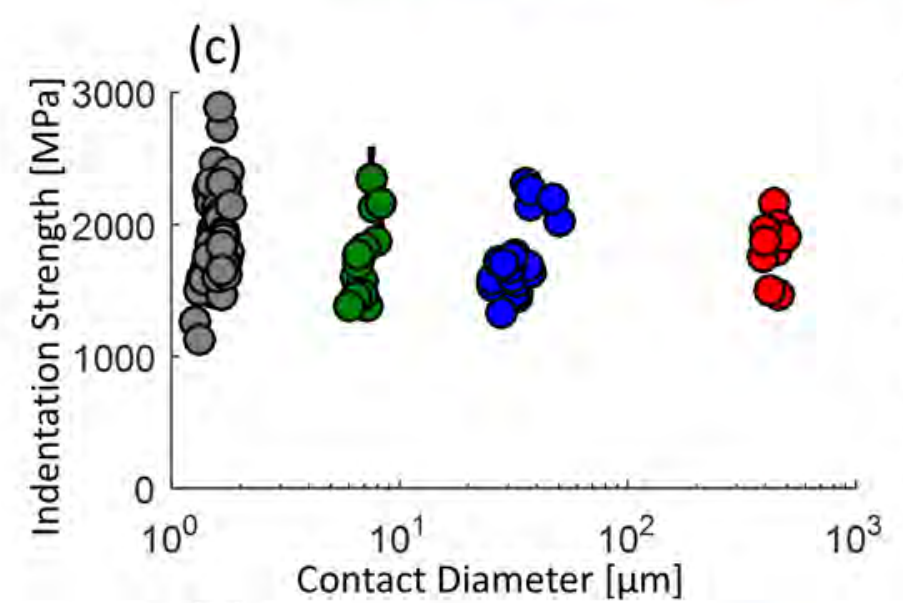

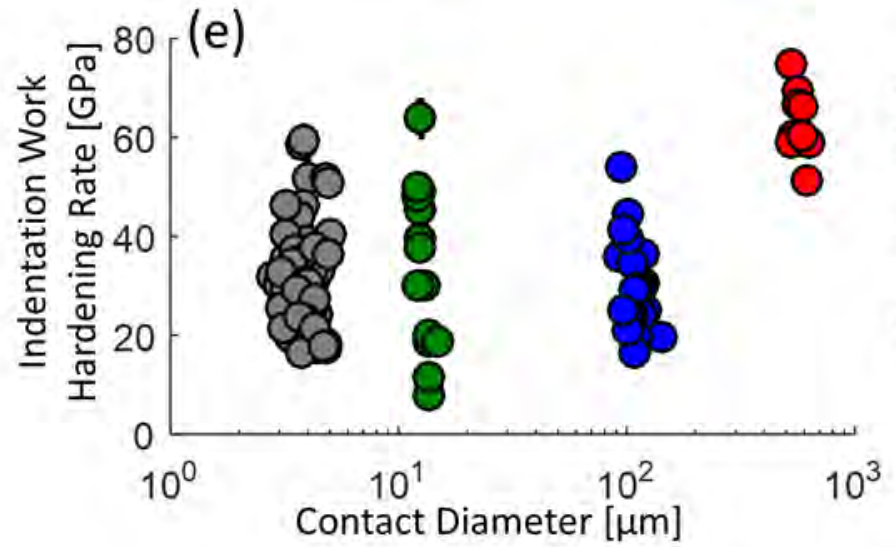

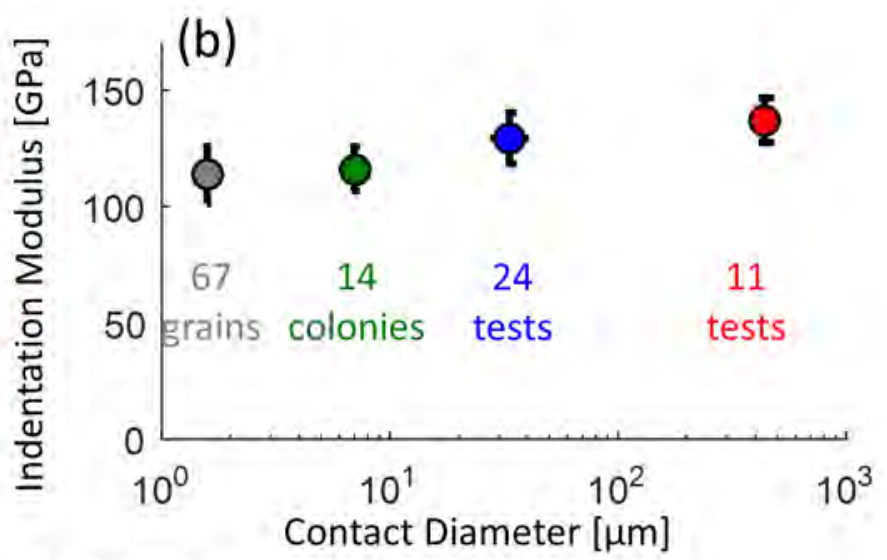
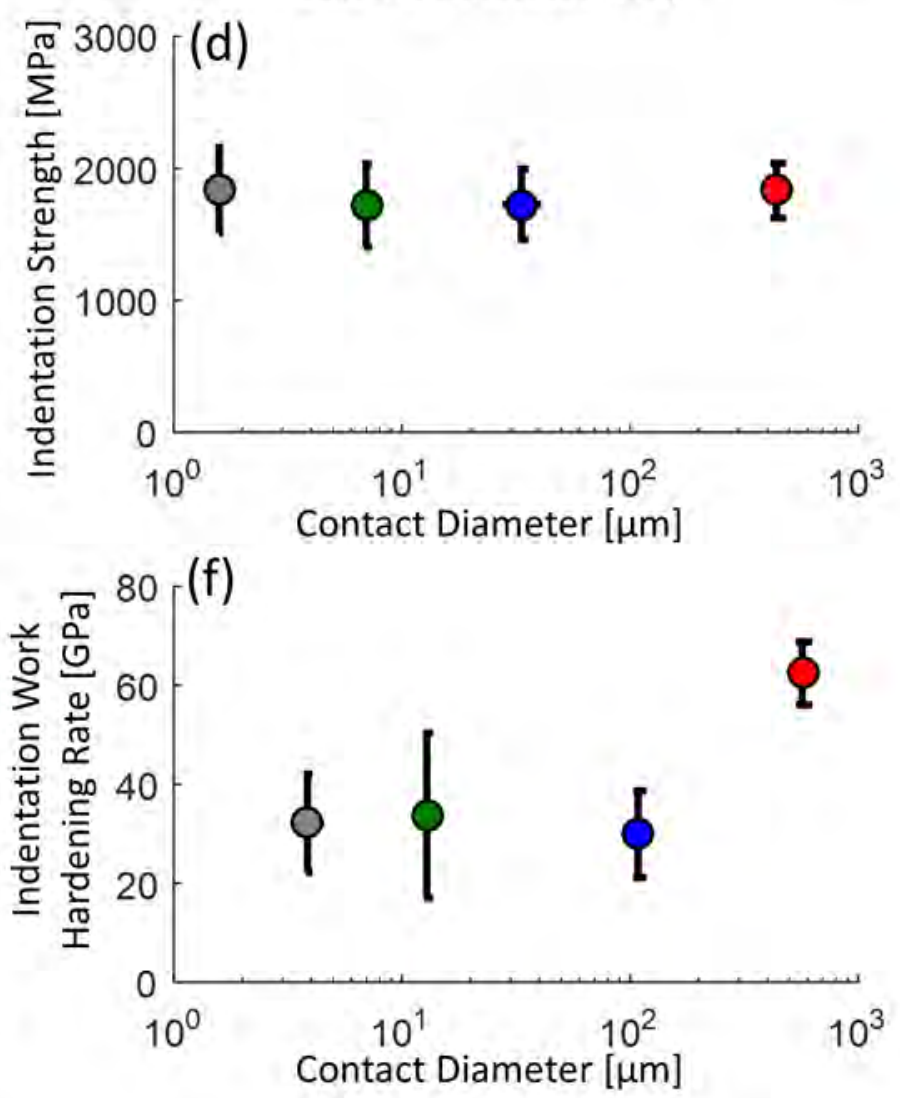


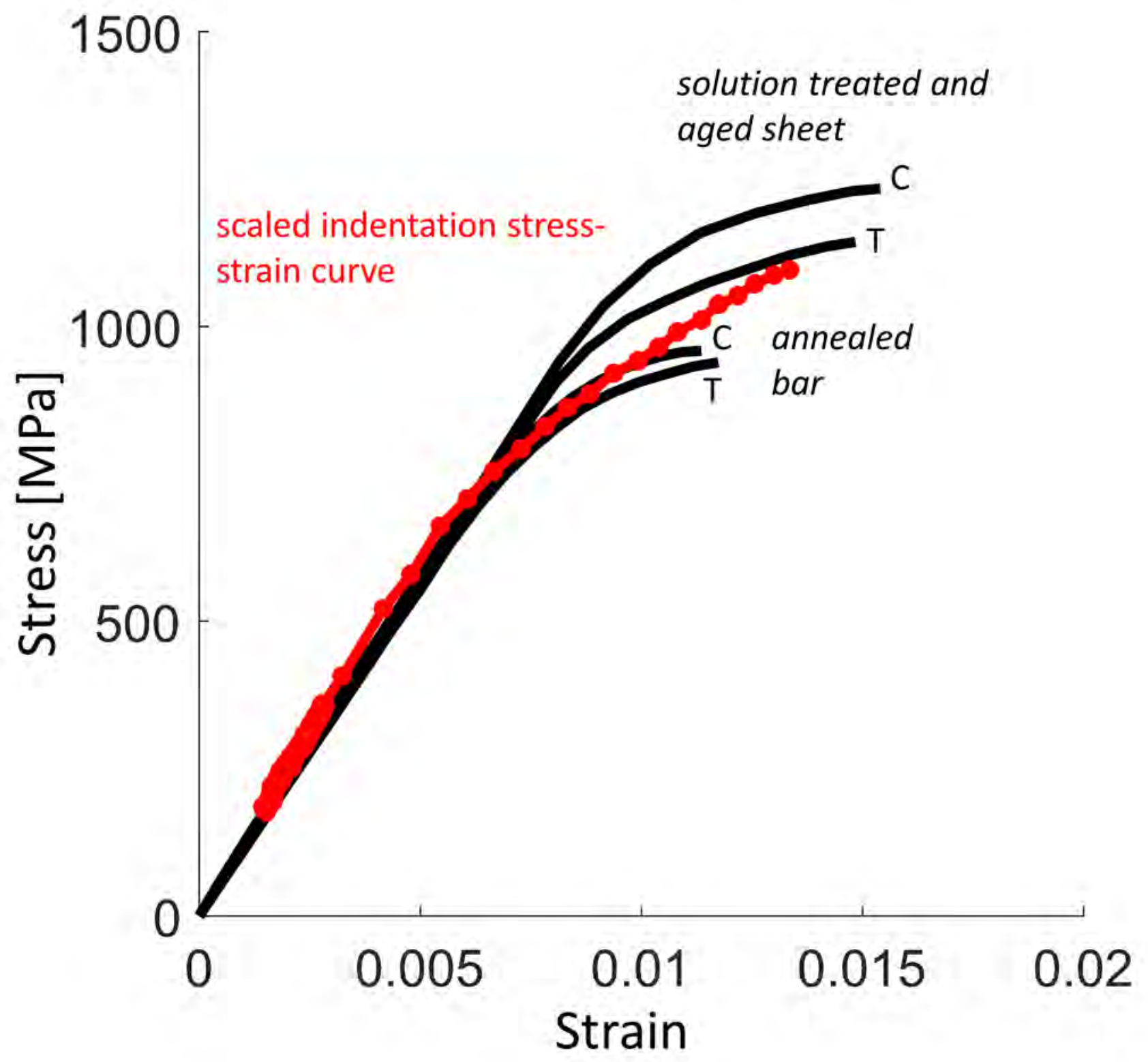

Figure 8. 

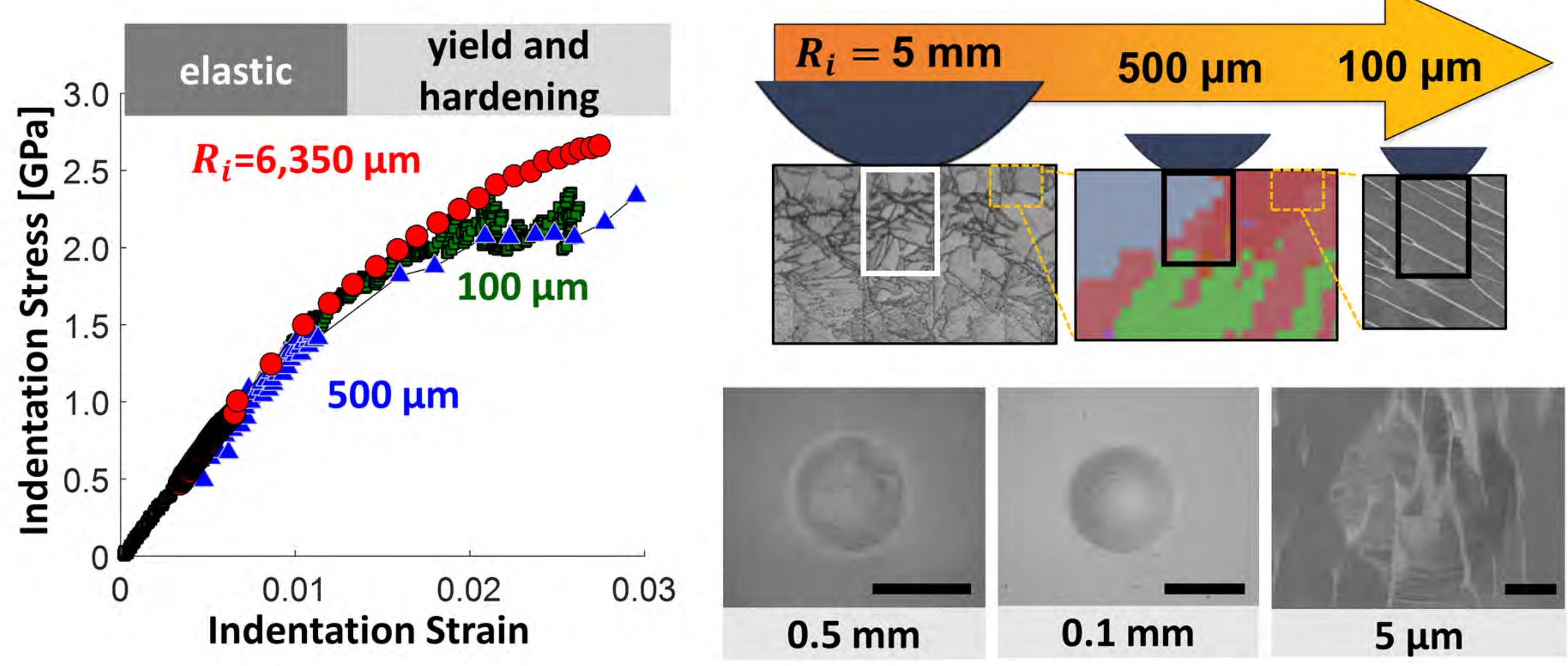